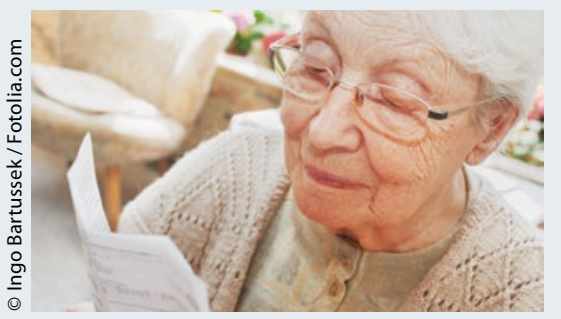

\section{Bundesrat macht sich für bessere} Beipackzettel stark

Beipackzettel sind für viele Patienten böhmische Dörfer. Der Bundesrat hat nun den Entschluss gefasst, die Medikamenteninformationen für Patienten und Verbraucher verständlicher zu gestalten: „Der Bundesrat bittet die Bundesregierung, sich auch weiterhin auf europäischer Ebene dafür einzusetzen, dass die Lesbarkeit der Packungsbeilagen von zentral oder dezentral in mehreren Mitgliedstaaten zugelassenen Arzneimitteln für Patientinnen und Patienten verbessert wird."

Christoph Winnat

\section{DAK startet virtuelle Sprechstunde}

Die DAK-Gesundheit bietet ihren Versicherten jetzt eine virtuelle Sprechstunde an, die alle Facharztgruppen und alle Diagnosegebiete umfassen soll. Der Service ist für DAK-Mitglieder kostenfrei über die Website der Krankenkasse erreichbar. Allerdings geht es nicht darum, dass die Patienten mit einem Wunscharzt oder ihrem Haus- beziehungsweise Facharzt kommunizieren können. Versicherte können vielmehr einen oder mehrere Wunschtermine für den Videokontakt wählen und angeben, welches Thema sie besprechen möchten. Kritik kommt zum Teil aus der Berufsverbänden der Facharztgruppen.

Rebekka Höhl

\section{Fachärzte behandeln 71,2 Millionen Patienten}

Von den jährlich 517,9 Millionen Behandlungsfällen der niedergelassenen Vertragsärzte und Vertragspsychotherapeuten sind mehr als die Hälfte $(265,2$ Millionen) bei den Fachärzten angesiedelt. Pro Quartal behandeln sie in Praxis und Klinik gut 71,2 Millionen Patienten, berichtet der Spitzenverband Fachärzte Deutschlands e. V. (SpiFa). $93 \%$ der fachärztlich versorgten Fälle in Deutschland finden, so der SpiFa, im ambulanten Versorgungsbereich statt, nur $7 \%$ in Kliniken. bremse in Sachen GOÄ-Reform. Fachgesellschaften und Berufsverbände hatten seit Monaten dringend eine Einsichtnahme in die Details der Leistungsbewertungen und Legendierungen gefordert, aber bis zur Ablehnung des Entwurfs im Präsidium der BÄK nicht bekommen. Auf der Suche nach der Ursachenforschung sind mehrere Punkte zu nennen:

—Die BÄK hat - anders als die KBV und die KVen - keinerlei originäre Information über die Privatliquidation ihrer Ärzte. Abrechnungsdaten, vor allem Leistungsfrequenzen und ihre Entwicklung, die am Ende zusammen mit den Bewertungen die Ausgabenentwicklung bestimmen, sind bei den PKV-Unternehmen monopolisiert. Dass diese Information Macht ist, musste der BÄK-Vorstand letzte Woche bitter erfahren.

_ Jede Neubewertung - etwa Aufwertung von Beratungs- und Gesprächsleistungen, Abwertung von Technik, Abschöpfung von Rationalisierungs- gewinnen - zieht arztgruppenspezifische Umverteilungen nach sich. Das ist aus vielen EBM-Reformen bekannt. Davon wurde der Vorstand der BÄK offenbar kalt erwischt.

_Die Honorarabteilung der BÄK, lange Zeit ohne eigenen Dezernenten, besteht aktuell gerade einmal aus vier Köpfen (Assistenzpersonal nicht gerechnet). Im September 2014 wechselte Dr. Bernhard Rochell, ein ausgewiesener Gebührenordnungsspezialist vom Amt des BÄK-Hauptgeschäftsführers zurück in die KBV-Verwaltungsspitze. Seitdem durfte sich die BÄK den Experten zeitweilig „ausleihen“. Die Vakanz dauerte bis Januar 2015.

Ob der Ärztetag in Hamburg Ende Mai nun einen Ausblick auf die weiteren Verhandlungen zur neuen GOÄ und zu den politischen Möglichkeiten der BÄK geben wird, ist ungewiss. Die GOÄ ist bislang nicht als thematischer Schwerpunkt vorgesehen.

Helmut Laschet, Hauke Gerlof

\title{
BSG gegen Verzögerungstaktik bei Praxisnachfolge
}

\section{Die Nachbesetzung der eigenen Praxis mit einem Wunschbewerber ist unter Umständen nicht machbar. Welche Regeln gelten?}

$\mathrm{D}$ ie Nachbesetzung von Arztsitzen liegt fest in der Hand der Zulassungsausschüsse. Versuchen Ärzte, durch Verzögerungen Einfluss auf die Auswahl zu nehmen, kann das zum Verlust des Nachbesetzungsrechts führen, wie das Bundessozialgericht (BSG) entschied (Aktenzeichen B 6 KA 9/15 R).

Dem Kläger war seine vertragsärztliche Zulassung zum 31. Januar 2011 entzogen worden. Im November 2010 hatte er die Nachbesetzung beantragt. Obwohl mehrere Bewerbungen vorlagen, zog der Arzt seinen Nachbesetzungsantrag kurz vor der Sitzung des Zulassungsausschusses zurück. Ebenso verfuhr er mit einem zweiten Antrag. Im Juni 2011 stellte der Arzt einen dritten Antrag. Mit einem Bewerber einigte er sich auf die Übernahme. Im September 2011 stellte jedoch der
Zulassungsausschuss fest, mehr als sechs Monate nach Ende der vertragsärztlichen Zulassung bestehe keine „fortführungsfähige Praxis“ mehr. Auch der Berufungsausschuss lehnte eine dritte Ausschreibung ab. Vor dem BSG betonte die beigeladene $\mathrm{KV}$, der abgebende Arzt habe keinen Anspruch auf „Wunschbewerber“. Insbesondere dürfe er das Verfahren nicht verzögern, „bis der Kandidat kommt, der am meisten bezahlt“.

Das BSG bestätigte zunächst, dass nach den derzeitigen gesetzlichen Regelungen die Nachbesetzung auch dann nicht ausscheidet, wenn die Zulassung wegen Pflichtverletzungen des Arztes entzogen wurde. Maßgeblich für die Frage einer fortführungsfähigen Praxis ist in der Regel immer der Tag des Antrags.
Martin Wortmann 